\title{
Fiabilidad y validez para un cuestionario de satisfacción con la formación continuada en salud: el cuestionario de satisfacción del discente
}

\author{
Thomas Esposito, Francisco J. Muñoz-Castro, Manuel Herrera-Usagre, Mayte Periáñez-Vega
}

Introducción. La formación continuada es el proceso de enseñanza y aprendizaje activo que tiene lugar a lo largo de toda
la vida profesional. Valorar el grado de satisfacción con dicho proceso se presenta como una tarea necesaria. Si bien la
mayoría de cuestionarios que evalúan la satisfacción del alumnado contemplan las diferentes dimensiones del proceso
formativo como la metodología empleada, la adecuación de los contenidos o la calidad de los docentes, no todos han
comprobado la fiabilidad y validez de los mismos. El objetivo del estudio es observar las características psicométricas del
cuestionario de satisfacción del discente (CSD) enmarcado en la herramienta 'eValúa' de la línea de proyectos para el de-
sarrollo profesional continuo de la Agencia de Calidad Sanitaria de Andalucía, España.

Sujetos y métodos. Análisis estadísticos de las puntuaciones del CSD $(n=1.628)$, usando análisis de fiabilidad, análisis de componentes principales y correlaciones de los factores.

Resultados. El CSD fue muy fiable, con un $\alpha$ de Cronbach global de 0,979 . Las dimensiones del cuestionario mostraron cargas factoriales elevadas (Utilidad: $R=85,9 ; \alpha=0,91$. Metodología: $R=77,4 ; \alpha=0,95$. Organización y recursos: $R=$ 73,25; $\alpha=0$,92. Capacidad docente: $R=90 ; \alpha=0,97$. Valoración global: $R=96,6 ; \alpha=0,96)$. Las dimensiones 'utilidad' y 'metodología' fueron las que presentaron niveles de correlación más altas con la totalidad del cuestionario y con las dos preguntas de valoración global con la formación.

Conclusiones. El CSD se presenta como una herramienta fiable y válida para la medición de la satisfacción con la formación continuada en salud. Dadas las altas correlaciones ítem-total que presentaron algunos ítems, se propone repasar su diseño para evitar concurrencias entre preguntas.

Palabras clave. Aprendizaje. Fiabilidad y validez. Formación continuada. Psicometría. Satisfacción.

Reliability and validity of a continuous training satisfaction questionnaire in health: the Learning Satisfaction Questionnaire

Introduction. Continuing training is conceived as the learning and permanent asset process to which all health professionals are entitled and obliged. Although most questionnaires that assess participants satisfaction look at the different dimensions of the learning process, such as methodology used, content appropriateness or teachers performance quality, not all have proven its reliability and validity. The aim of the study is to observe the psychometric characteristics of Teaching Satisfaction Questionnaire (CSD) framed in the evaluation tool 'eValúa', designed by the Continuing Professional Development Project of the Andalusian Agency for Healthcare Quality (Spain).

Subjects and methods. Statistical analysis of the scores of CSD with a sample of 1628 cases using reliability analysis, principal component analysis, and correlation of the factors that are derived from the five dimensions that make up the questionnaire.

Results. The CSD was highly reliable with an overall Cronbach's $\alpha$ of 0.979 . The dimensions of the questionnaire showed high factor loadings (Utility: $R=85.9 ; \alpha=0.91$. Methodology: $R=77.4 ; \alpha=0.95$. Organization and resources: $R=73.25$; $\alpha=0.92$. Teaching capacity: $R=90 ; \alpha=0.97$. Global satisfaction: $R=96.6 ; \alpha=0.96)$. The 'utility' and 'methodology' dimensions were those that showed the highest levels of correlation with both the entire questionnaire and the two global satisfaction questions.

Conclusions. The CSD is presented as a reliable and valid tool for measuring satisfaction with continuing education in health. Given the high item-total correlations some items showed, a review of the questionnaire design is proposed to avoid concurrency between questions. Future analysis of the differences in satisfaction with training according to different socio-professional characteristics was raised.

Key words. Continuous education. Psychometrics. Satisfaction. Reliability and validity.
Agencia de Calidad Sanitaria de Andalucía; Fundación Progreso y Salud (T. Esposito, F.J. Muñoz-Castro, M. Herrera-Usagre, M. PeriáñezVega). Departamento de Sociología; Universidad de Sevilla (M. HerreraUsagre). Sevilla, España.

Correspondencia:

Dr. Manuel Herrera Usagre. Agencia de Calidad Sanitaria de Andalucía. Augusto Peyré, 1. Edificio Olalla, 3. a planta. E-41020 Sevilla.

Fax:

+34955023901.

E-mail:

manuel.herrera.usagre@ juntadeandalucia.es

Conflicto de intereses: No declarado.

Competing interests: None declared.

(C) 2015 FEM 


\section{Introducción}

La formación continuada es el proceso de enseñanza y aprendizaje activo que tiene lugar a lo largo de toda la vida profesional. Tiene como objetivo actualizar y mejorar las competencias de los profesionales de la salud ante la evolución científica, tecnológica y organizativa, así como atender a las demandas y necesidades, tanto sociales como del propio sistema sanitario. Esta definición engloba los conceptos de formación continuada y desarrollo profesional continuo, entendiéndose por formación continuada el aprendizaje de las personas que ya están desempeñando su práctica profesional [1-4].

Con el objetivo de asegurar la calidad con la formación recibida, se han desarrollado numerosos cuestionarios tanto para profesionales sanitarios en periodo de grado como de posgrado [5-10]. LozanoLozano et al [9] señalan la dificultad de encontrar un método ideal de medir la satisfacción con la formación recibida, en su caso de estudio, en la formación de grado. No obstante, remarcan el hecho de que la utilidad de este tipo de mediciones depende fuertemente de la cooperación del factor humano en el proceso. La 'satisfacción con la formación recibida' la definen como un concepto multidimensional donde diferentes medidas deben tenerse en cuenta [9]. Para poder sintetizar la satisfacción con la formación recibida en un único constructo -se tenga o no la intención de condensarlo en un único índice compuesto-, el diseño de sus dimensiones debe basarse en un riguroso marco teórico y conceptual [11]. Con su cuestionario de 19 ítems, Lozano-Lozano et al elaboraron un índice compuesto de 'satisfacción con la formación recibida' a partir de siete dimensiones, cinco relacionadas con el proyecto formativo (objetivos y contenidos, metodología y clima, personal docente, evaluación, organización y gestión externa) y otras dos con la satisfacción global (utilidad; participación, acción y actitud). Del mismo modo, la mayoría de cuestionarios basan su evaluación fundamentalmente en los contenidos, la calidad docente, los criterios de evaluación y la satisfacción global [6]. Otros, como el Postgraduate Hospital Educational Environment Measure (PHEEM), dividen 40 ítems en sólo tres dimensiones: autonomía, calidad docente y apoyo social del docente $[8,12]$.

En mayo de 2012, la línea de proyectos para el desarrollo profesional de la Agencia de Calidad Sanitaria de Andalucía (ACSA) elaboró un cuestionario de satisfacción del discente (CSD) con el objetivo de, por un lado, garantizar la calidad del diseño de esas actividades y programas [13] y, por otro, facilitar las tareas de evaluación a los profesionales que desarrollan labores de gestión de la formación continuada en salud. El cuestionario es una herramienta de evaluación que contempla 23 ítems en cinco dimensiones (utilidad, metodología, organización y recursos, capacidad docente, valoración global). Este cuestionario se encuadra dentro de la herramienta 'eValúa' que permite la gestión descentralizada y autónoma de la evaluación de las actividades de formación, facilitando la planificación del envío de los cuestionarios para su cumplimentación, así como la recogida y análisis de los resultados [13,14].

El CSD se ha elaborado teniendo en cuenta los criterios establecidos en los programas de acreditación de la ACSA [13]. El objetivo del presente artículo es evaluar la fiabilidad de consistencia interna y la validez de constructo del CSD (dadas las similitudes con el cuestionario desarrollado por autores como Lozano-Lozano et al [8] y el propio método de construcción del cuestionario basado en otros ya implementados, no creímos pertinente realizar un análisis de validez de contenido).

\section{Sujetos y métodos}

\section{Diseño del estudio}

El estudio tiene un diseño psicométrico. Para asegurar la validez de las escalas se procedió en dos fases: pruebas psicométricas de fiabilidad de consistencia interna ( $\alpha$ de Cronbach) y validez de constructo (análisis de componentes principales).

\section{Diseño del cuestionario}

En la elaboración del CSD han intervenido profesionales expertos en calidad de la formación, pertenecientes a la ACSA y a las unidades de formación o gestión del conocimiento de otras entidades del Sistema Sanitario Público de Andalucía. Como tarea previa, se analizaron varios cuestionarios empleados para evaluar la satisfacción de los participantes en actividades formativas en el ámbito de la salud, y en base a esa información se diseñaron las dimensiones y se elaboraron los ítems. Todos los ítems están recogidos en una escala donde 0 es el 'grado más bajo de satisfacción o estar totalmente en desacuerdo', y 10, el 'grado más alto de satisfacción o estar totalmente de acuerdo'.

\section{Participantes}

La población del estudio está formada por profesionales de la salud que asistieron a alguna actividad 
formativa desde mayo de 2012 a junio de 2013 en algún centro de la región de Andalucía (la aplicación también está disponible de manera libre para otros centros y programas de formación en salud de cualquier parte del mundo, pero su incidencia es menor del $2 \%$ de los casos). Se han seleccionado la totalidad de sujetos que respondieron voluntariamente al CSD elaborado por el Observatorio para la Calidad de la Formación Continuada. En total, se recogieron 1.667 respuestas, de las cuales finalmente se utilizaron 1.628 una vez se eliminaron los casos con algún ítem sin respuesta.

\section{Procedimiento y recogida de los datos}

El CSD es utilizado por los profesionales responsables de la actividad formativa. Estos profesionales son los encargados de distribuir los cuestionarios a los y las discentes, en soporte papel o digital. La evaluación ha de realizarse a partir del día en que finaliza la actividad formativa, condición que la herramienta 'eValúa' aplica para activar el enlace correspondiente a la versión web del cuestionario. A partir de esa fecha, el responsable de formación o coordinador de la actividad formativa envía un correo desde la aplicación a todos los discentes con la información necesaria para cumplimentar el cuestionario: enlace web, plazo, datos de la actividad, finalidad de la encuesta y normas de privacidad. De manera alternativa, puede imprimir el cuestionario y entregarlo en mano al alumnado. Para el cálculo de la duración se ha tomado la fecha y la hora en la que se ha empezado y terminado la cumplimentación del cuestionario. Así, se ha obtenido el tiempo empleado en su cumplimentación. Finalmente, se ha obtenido la media de este periodo temporal (se han ignorado del cálculo aquellos registros donde ambas fechas y horas son iguales, ya que es imposible que transcurran cero segundos, así como aquellos registros en los que el tiempo invertido supera las cinco horas). Con las respuestas guardadas y aplicando los criterios anteriormente descritos, a fecha de agosto de 2013, se obtiene un tiempo medio de 6 minutos y 22 segundos.

\section{Mediciones psicométricas}

\section{Consistencia interna y homogeneidad}

Se calculó el $\alpha$ de Cronbach para estimar la consistencia interna del cuestionario. La consistencia interna debe ser condición necesaria para la homogeneidad o unidimensinalidad de una escala, tomando como criterio de fiabilidad un valor $\alpha>0,7$ $[15,16]$.

\section{Validez de constructo}

Los datos se trataron usando un análisis factorial (análisis de componentes principales sin rotación). Para alcanzar el mejor ajuste de la estructura y el menor número de factores, se emplearon los siguientes criterios: eigenvalues $>1,0$ y cargas factoriales $>0,40$. Antes de comenzar los análisis, se realizaron los tests de esfericidad de Bartlett y el test KaiserMeyer-Olkin (KMO) con el fin de comprobar si el tamaño de la muestra es lo suficientemente grande para ejecutar un análisis factorial (KMO > 0,5; $p<$ 0,05 para el test de Bartlett), siendo los resultados satisfactorios.

\section{Consideraciones éticas}

Se obtuvo la autorización de la ACSA, entidad responsable de la aplicación 'eValúa', para el análisis de los datos con propósitos estrictamente científicos. El anonimato y la confidencialidad de los datos aportados por los participantes se garantizaron y especificaron en el propio cuestionario.

\section{Resultados}

\section{Consistencia interna y homogeneidad}

Los análisis mostraron que el CSD tiene un $\alpha$ de Cronbach global de 0,979. El cálculo del test de Bartlett y del test KMO mostraron un valor de 0,969 y un valor de $p<0,001$, indicando que la muestra era lo suficientemente grande para implementar satisfactoriamente un análisis factorial. Del análisis de componentes principales con el total del cuestionario se obtuvieron dos factores (en lugar de los cinco en los que se divide el cuestionario), con eigenvalues $>1,0$ y un $75,83 \%$ de varianza explicada acumulada, lo que demuestra la alta correlación entre preguntas y dimensiones. Las cargas factoriales de todos los ítems fueron satisfactorias, con un rango de 0,812-0,983 (Tabla I).

\section{Validez de constructo}

Seguidamente, se implementaron una serie de análisis para cada una de las subdimensiones del cuestionario (utilidad, metodología, organización y recursos, capacidad docente, valoración global) (Tabla I). Se calculó un solo factor interno para cada una de las dimensiones. Todas las dimensiones presentaron altos porcentajes de varianza explicada, así como puntuaciones excelentes de $\alpha$ de Cronbach. Para el primer factor (utilidad), el $\alpha$ de Cronbach fue 
Tabla I. Análisis de componentes principales y análisis de $\alpha$ de Cronbach.

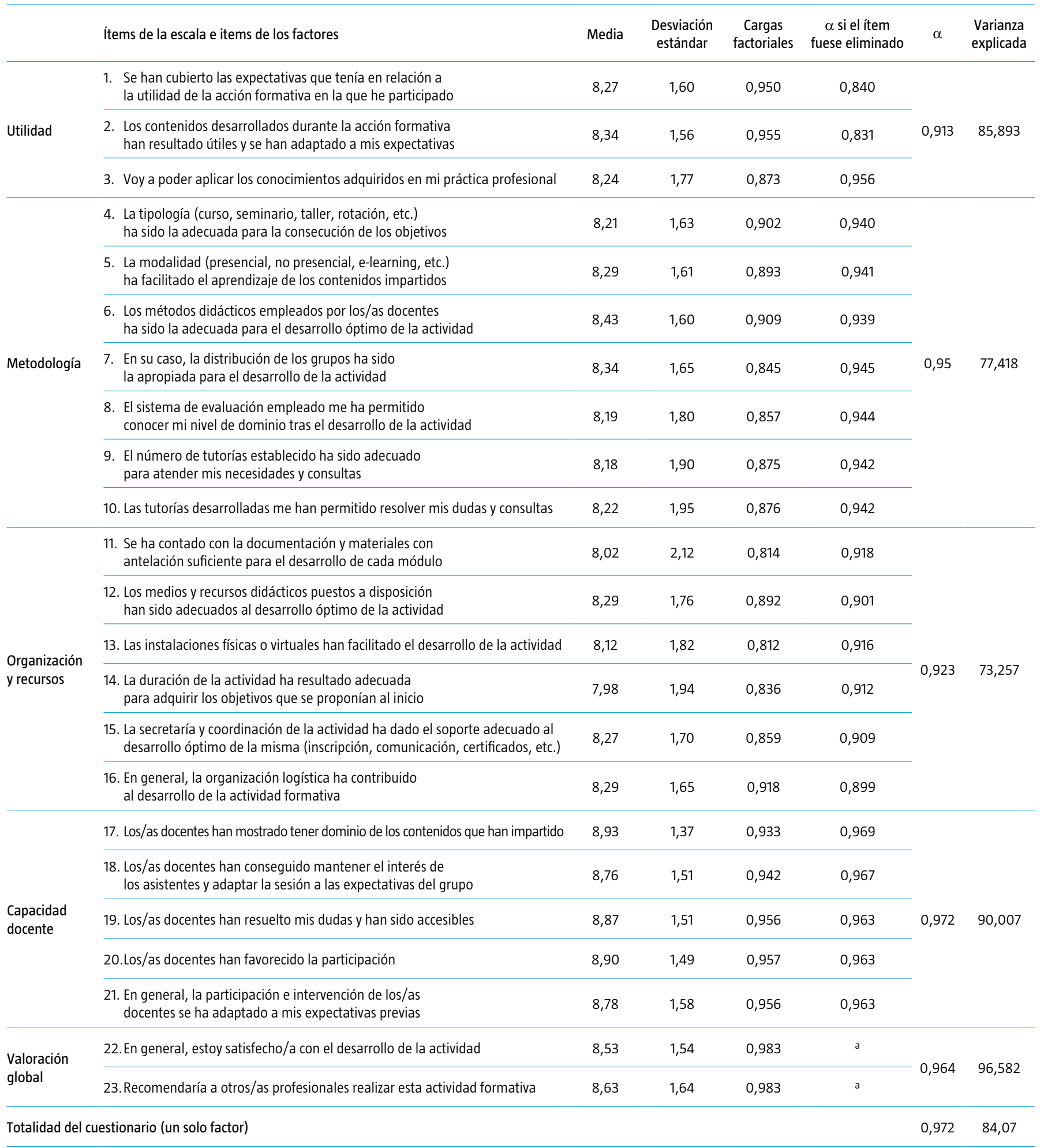

a Dado que la dimensión sólo tiene dos ítems, este parámetro no ha podido calcularse. 
Tabla II. Matriz de correlaciones entre los factores, el cuestionario y preguntas sobre evaluación global.

\begin{tabular}{|c|c|c|c|c|c|c|c|c|}
\hline & Utilidad & Metodología & $\begin{array}{c}\text { Organización } \\
\text { y recursos }\end{array}$ & $\begin{array}{l}\text { Capacidad } \\
\text { docente }\end{array}$ & $\begin{array}{l}\text { Valoración } \\
\text { global }\end{array}$ & Cuestionario & Ítem 22 & Ítem 23 \\
\hline Utilidad & 1 & & & & & & & \\
\hline Metodología & $0,870^{a}$ & 1 & & & & & & \\
\hline Organización y recursos & $0,750^{a}$ & $0,841^{a}$ & 1 & & & & & \\
\hline Capacidad docente & $0,724^{a}$ & $0,809^{a}$ & $0,693^{a}$ & 1 & & & & \\
\hline Valoración global & $0,859^{a}$ & $0,901^{a}$ & $0,901^{\mathrm{a}}$ & $0,901^{a}$ & 1 & & & \\
\hline Cuestionario & $0,897^{a}$ & $0,966^{a}$ & $0,907^{a}$ & $0,891^{a}$ & $0,870^{a}$ & 1 & & \\
\hline Ítem 22 & $0,855^{a}$ & $0,860^{a}$ & $0,790^{a}$ & $0,801^{a}$ & $0,855^{a}$ & $0,904^{a}$ & 1 & \\
\hline Ítem 23 & $0,834^{a}$ & $0,832^{a}$ & $0,743^{a}$ & $0,794^{a}$ & $0,834^{a}$ & $0,876^{a}$ & $0,932^{a}$ & 1 \\
\hline
\end{tabular}

de 0,91 y los tres ítems de los que se compone lograban explicar un $85,9 \%$ de la varianza. El segundo factor (metodología) presentó un $\alpha$ de Cronbach de 0,95 y los ítems mostraban una varianza explicada de un $77,4 \%$. El tercer factor (organización y recursos) obtuvo un $\alpha$ de Cronbach de 0,92 y el conjunto de sus ítems lograron explicar un $73,2 \%$ de la varianza del factor. El cuarto factor (capacidad docente) logró explicar un $90 \%$ de su varianza y obtuvo un alfa de Cronbach de 0,97. Finalmente, los ítems del último factor (valoración global) lograron explicar un $96,5 \%$ de su varianza y obtuvieron un $\alpha$ de Cronbach de 0,96 . En la tabla II se muestra la matriz de correlaciones entre las dimensiones, un factor compuesto con la totalidad del cuestionario y las dos preguntas de valoración global de la actividad formativa (ítems 22 y 23). Los resultados ponen en evidencia la importancia de las evaluaciones sobre la metodología, dado que presenta el nivel de correlación más alto con la totalidad de las preguntas. Lograr ver la utilidad de los contenidos y estar satisfecho con la metodología empleada son factores clave de cara a la satisfacción general con el desarrollo de la actividad formativa.

\section{Discusión}

El propósito de este estudio era validar el CSD como herramienta de evaluación de la formación continuada en salud, atendiendo a la opinión de los par- ticipantes ante diferentes dimensiones del desarrollo de las actividades formativas. Medir la fiabilidad y la validez de este instrumento de evaluación se hace vital para poder comprobar su utilidad para los formadores y crear un ambiente de aprendizaje eficaz en el entorno de la salud $[15,16]$. La utilización del $\alpha$ de Cronbach para los análisis de fiabilidad de consistencia interna y del análisis de componentes principales para la validez de constructo viene avalada por las revisiones de la bibliografía [17].

Nuestros resultados evidenciaron un alto nivel de consistencia interna del instrumento, tal y como habían averiguado otros estudios sobre cuestionarios con los mismos propósitos [5-8]. El análisis de componentes principales para cada una de las cinco dimensiones en las que se compone el CSD confirmó su validez de constructo. Cada una de las dimensiones mostró porcentajes de varianza explicada del 73-96\%. Igualmente, las correlaciones ítemtotal, que en nuestro caso coinciden con las cargas factoriales porque no se aplicó ningún tipo de rotación en el análisis factorial, se mostraron en todos los ítems superiores a 0,87. Cuando abordamos las correlaciones de cada una de las dimensiones con la totalidad del cuestionario se evidenció la importancia de las dimensiones 'utilidad' y 'metodología'. Ambas dimensiones fueron también las que mostraron una mayor correlación con las dos preguntas sintéticas de evaluación global de la actividad formativa (ítems 22 y 23). Este hecho pone de manifiesto la importancia de realizar un adecuado diseño de la acti- 
vidad formativa, contemplando especialmente la descripción de su utilidad, y de las herramientas metodológicas y didácticas que van a emplearse para entregar los contenidos.

Aunque las dimensiones 'organización y recursos' y 'capacidad docente' también mostraron altas correlaciones, sus puntuaciones fueron ligeramente menores, lo que se traduce en un mayor margen de mejora. No obstante, estos resultados siguen siendo congruentes con los obtenidos por Vieira [7] y Aspegren et al [18], quienes encontraron los aspectos relacionados con los docentes como los más importantes en la medición del ambiente educacional, a tenor de las correlaciones halladas. Por otro lado, las puntuaciones medias de las valoraciones en este estudio se adecuan a los niveles de satisfacción con otros programas de desarrollo profesional continuo y formación continuada [19-21]. La importancia de medir regularmente estos aspectos es vital a la hora de introducir cambios en los programas formativos, adaptándolos tanto a los requerimientos de las organizaciones como a las necesidades de los participantes [22].

Son notables los estudios recientes que avalan el efecto positivo de la formación continuada en el desarrollo de las competencias de los profesionales de la salud de cara a ejercer una práctica profesional basada en la evidencia [23-25]. El análisis aquí aportado ofrece la validez y fiabilidad necesarias a cualquier cuestionario que tenga como objetivo proveer a los organizadores y formadores de un feedback sistemático por parte los participantes en las actividades formativas [26].

\section{Aplicaciones prácticas}

Las puntuaciones excesivas del $\alpha$ de Cronbach que muestra el propio cuestionario y, en particular, la dimensión 'capacidad docente' ( $\alpha$ de Cronbach $>0,95$ ), indican redundancia en algunas preguntas que podrían simplificarse eventualmente en versiones futuras, tal y como recomienda la bibliografía [27]. La capacidad docente es uno de los factores que más influye en la creación de un ambiente de aprendizaje adecuado $[6,9]$. Así, en versiones del CSD que se encuentran en desarrollo, se está contemplando la necesidad de desagregar los ítems de esta dimensión para que cada uno de los docentes de la actividad formativa sea evaluado de manera independiente, en lugar de una evaluación conjunta. Por otro lado, el análisis de componentes principales con la totalidad del cuestionario ofrece conclusiones idénticas a las obtenidas por Boor et al [28] y Vieira [7] en sus análisis de validez para cuestionarios de me- dición del ambiente educacional. Nuestros resultados han confirmado también una tendencia a la unidimensionalidad de todo el cuestionario, dado que se obtuvieron sólo dos dimensiones con eigenvalues $>1,0$ en lugar de las cuatro que esperaríamos según la estructura del cuestionario, pudiendo el primer factor obtenido explicar por sí solo el 69,8\% de la varianza. Este hecho plantea la conveniencia de presentar las preguntas del cuestionario sin organizarlas por bloques, lo que permitiría una reflexión más efectiva por parte del discente.

\section{Limitaciones}

El CSD tiene como objetivo ofrecer una herramienta de evaluación para los gestores de la formación. Dado que en las actividades de formación continuada el número de participantes no es amplio [23], se omiten numerosos datos (edad, titulación, especialidad, años trabajando en el sistema de salud, etc.) para garantizar la confidencialidad y la sinceridad de la información aportada. No obstante, en próximas versiones del cuestionario se ofrecerá aportar voluntariamente esta información para poder analizar si existen diferencias en los niveles de satisfacción en función de estos factores. Del mismo modo, en la próxima versión se incluirá la realización de un análisis de fiabilidad test-retest para una muestra de casos.

\section{Bibliografía}

1. Muñoz-Castro FJ, Valverde-Gambero E, Villanueva-Guerrero L, Mudarra-Aceituno MJ, Vázquez-Vázquez M, Almuedo-Paz A. Evolución de la formación continuada acreditada tras la puesta en marcha de la estrategia para la seguridad del paciente. Revista de Calidad Asistencial 2012. URL: http://linkinghub. elsevier.com/retrieve/pii/S1134282X12000139. [30.07.2012].

2. Periáñez-Vega M. La evaluación del impacto de la formación. 2013. URL: http://www.slideshare.net/observatorioformacion/ la-evaluacin-del-impacto-de-la-formacin. [02.03.2014].

3. Ministerio de Sanidad, Política Social e Igualdad. Proyecto de real decreto por el que se determinan y clasifican las especialidades en ciencias de la salud y se desarrollan determinados aspectos del sistema de formación sanitaria especializada. Madrid; 2003.

4. Cantillon P, Jones R. Does continuing medical education in general practice make a difference? BMJ 1999; 318: 1276-9.

5. Bierer SB, Fishleder AJ, Dannefer E, Farrow N, Hull AL. Psychometric properties of an instrument designed to measure the educational quality of graduate training programs. Eval Health Prof 2004; 27: 410-24.

6. Herrera CA, Olivos T, Román JA, Larraín A, Pizarro M, Solís N, et al. Evaluation of the educational environment in medical specialty programs. Rev Med Chil 2012; 140: 1554-61.

7. Vieira JE. The Postgraduate Hospital Educational Environment Measure (PHEEM) questionnaire identifies quality of instruction as a key factor predicting academic achievement. Clinics 2008. URL: http://www.scielo.br/scielo.php?script=sci_arttext\&pid= S1807-59322008000600006\&lng=en\&nrm=iso\&tlng=en. [03.02.2014].

8. Gooneratne IK, Munasinghe SR, Siriwardena C, Olupeliyawa AM, Karunathilake I. Assessment of psychometric properties of a 
modified PHEEM questionnaire. Ann Acad Med Singap 2008; 37: 993-7.

9. Lozano-Lozano JA, Sanduvete-Chaves S, Chacón-Moscoso S, Pérez-Gil JA. Elaboration of an instrument to measure the construct 'satisfaction with the training received. International Journal of Social Science Studies 2013. URL: http://www. redfame.com/journal/index.php/ijsss/article/view/142. [03.02.2014].

10. Sanduvete-Chaves S, Holgado-Tello FP, Chacón-Moscoso S, Barbero-García MI. Measurement invariance study of the Training Satisfaction Questionnaire (TSQ). Span J Psychol 2013; 16: E28.

11. OCDE, Joint Research Center. Composite Indicators. 2008.

12. Wall D, Clapham M, Riquelme A, Vieira J, Cartmill R, Aspegren K, et al. Is PHEEM a multi-dimensional instrument? An international perspective. Med Teach 2009; 31: e521-7.

13. Agencia de Calidad Sanitaria de Andalucía. Programa integral para la acreditación de la formación continuada de las profesiones sanitarias unidades y centros de formación continuada, programas de actividades de formación continuada, actividades de formación continuada. Sevilla: ACSA; 2008.

14. Agencia de Calidad Sanitaria de Andalucía. Programa de desarrollo profesional continuo. 2013. URL: http:// formacionsalud.acsa.junta-andalucia.es/agenciadecalidadsanitaria/ formacionsalud/microsite/cms/es/evalua. [02.03.2014]

15. Clark LA, Watson D. Constructing validity: basic issues in objective scale development. Psychol Assess 1995; 7: 309-19.

16. Yurdugül H. Minimum sample size for Cronbach's coefficient alpha: a Monte Carlo Study. HU Journal of Education 2008; 35: 397-405.

17. Wetzel AP. Factor analysis methods and validity evidence: a review of instrument development across the medical education continuum. Acad Med 2012; 87: 1060-9.

18. Aspegren K, Bastholt L, Bested KM, Bonnesen T, Ejlersen E, Fog I, et al. Validation of the PHEEM instrument in a Danish hospital setting. Med Teach 2007; 29: 498-500.
19. Curran V, Sargeant J, Hollett A. Evaluation of an interprofessional continuing professional development initiative in primary health care. J Contin Educ Health Prof 2007; 27: 241-52.

20. Almuedo-Paz A, Brea-Rivero P, Buiza-Camacho B, Rojas-De Mora Figueroa A, Torres-Olivera A. Utilidad de la acreditación de competencias profesionales en el desarrollo profesional continuo. Revista de Calidad Asistencial 2011; 26: 221-7.

21. Ellencweig N, Weizman A, Fischel T. Factors determining satisfaction in psychiatry training in Israel. Acad Psychiatry 2009; 33: 169-73.

22. López-Rodrigo M. The evaluation of continuous training in healthcare organisations. A bibliographic review. Rev Enferm 2012; 35: 22-6, 29-34.

23. Liabsuetrakul T, Sirirak T, Boonyapipat S, Pornsawat P. Effect of continuous education for evidence-based medicine practice on knowledge, attitudes and skills of medical students. J Eval Clin Pract 2013; 19: 607-11.

24. Deljo E, Babacic R, Meskovic B, Grabus J. Impact of continuous education about oral hygiene on the changes of Cpi-index for 12-15-year-old schoolchildren. Acta Informatica Medica 2011; 19: 220.

25. Kulier R, Gülmezoglu AM, Zamora J, Plana MN, Carroli G, Cecatti JG, et al. Effectiveness of a clinically integrated e-learning course in evidence-based medicine for reproductive health training: a randomized trial. JAMA 2012; 308: 2218-25.

26. Rattray J, Jones MC. Essential elements of questionnaire design and development. J Clin Nurs 2007; 16: 234-43.

27. Streiner DL. Starting at the beginning: an introduction to coefficient alpha and internal consistency. J Pers Assess 2003; 80: 99-103.

28. Boor K, Scheele F, Van der Vleuten CPM, Scherpbier AJJA, Teunissen PW, Sijtsma K. Psychometric properties of an instrument to measure the clinical learning environment. Med Educ 2007; 41: 92-9. 